\title{
Avaliação da sobrecarga dos cuidadores de pessoas com Esclerose Lateral Amiotrófica (ELA) ${ }^{1}$
}

\author{
Lilian Maria Sanguinett de Almeidaa ${ }^{a}$ Ilka Veras Falcáo ${ }^{b}$, Tatiana Lins Carvalhoc \\ ${ }^{\mathrm{a} U n i v e r s i d a d e ~ F e d e r a l ~ d e ~ P e r n a m b u c o ~-~ U F P E, ~ R e c i f e, ~ P E, ~ B r a s i l . ~}$ \\ bepartamento de Terapia Ocupacional, Universidade Federal de Pernambuco - UFPE, Recife, PE, Brasil. \\ 'Hospital Universitário Oswaldo Cruz, Universidade de Pernambuco - UPE, Recife, PE, Brasil.
}

\begin{abstract}
Resumo: Introdução: Esclerose lateral amiotrófica (ELA) é uma desordem neurodegenerativa que resulta na morte seletiva de neurônios motores, causando fraqueza e atrofia muscular generalizada como principais sintomas. Ainda sem cura, o doente em tratamento requer assistência na mobilidade e atividades diárias. Cuidar de um paciente com ELA implica maior ou menor sobrecarga e tempo, demandando estratégias de redução do estresse e ampliação da qualidade de vida dos cuidadores. Nesse sentido, os grupos de apoio surgem como alternativas. Objetivo: O objetivo deste estudo é avaliar se há sobrecarga dos cuidadores de pacientes com ELA e os possíveis benefícios relacionados à participação no grupo de apoio específico a cuidadores. Método: Trata-se de um estudo descritivo e transversal, com aplicação da Escala de Sobrecarga do Cuidador - Zarit e entrevista sobre a participação no Grupo de Apoio Esperança para ELA, realizado em um hospital público do Recife. Foram avaliados sete cuidadores familiares e um profissional. Resultados: Após análise dos dados, verificou-se que a maioria dos cuidadores apresenta sobrecarga moderada e quase todos residem com o doente, o que pode contribuir para limitações e modificações no estilo de vida destes. A participação no grupo é reconhecida por todos como fonte de trocas e aprendizado sobre a doença, além de acolhedora de questões emocionais e dificuldades no cuidado. Conclusão: Conclui-se que esses cuidadores evidenciam sobrecarga moderada e se beneficiam do apoio recebido no Grupo Esperança para ELA. No entanto, outras estratégias podem ser necessárias para minimização dessa sobrecarga e segurança no cuidado ao doente.
\end{abstract}

Palavras-chave: Esclerose Amiotrófica Lateral, Cuidadores, Efeitos Psicossociais da Doença, Determinação de Necessidades de Cuidados de Saúde.

\section{Evaluation of overloading on caregivers of people with Amyotrophic Lateral Sclerosis (ALS)}

\begin{abstract}
Introduction: Amyotrophic lateral sclerosis (ALS) is a neurodegenerative disorder that results in the selective death of motor neurons, with weakness and generalized muscle atrophy as primary symptoms. With cure yet to be found, the patients in treatment need assistance in mobility and daily activities. Taking care of a patient with ALS results in varying degrees of burden and time, demanding stress reduction strategies and an increase in the quality of life of caregivers. In this sense, support groups emerge as alternatives. Objective: The aim of this study was to assess whether caregivers of patients with ALS exhibit burden and the possible benefits from participating in a support group specifically designed for them. Method: This is a cross-sectional descriptive study that applied the Zarit Caregiver Burden Scale and Interview regarding participation in the "Hope for ALS" support group, at a public hospital in Recife, Brazil. Seven families' caregivers and one professional caregiver were assessed. Results: Data analysis showed that most caregivers exhibit moderate burden and nearly all live with the patient, which may contribute to limitations in their lifestyle. Participation in the group is recognized by all as a source of
\end{abstract}

Autor para correspondência: Ilka Veras Falcão, Departamento de Terapia Ocupacional, Universidade Federal de Pernambuco, Av. Prof. Moraes Rego, 1235, Cidade Universitária, CEP 50670-901, Recife, PE, Brasil, e-mail: ilkafalcao.ufpe@gmail.com

Recebido em Jun. 4, 2016; $1^{\text {a }}$ Revisão em Jan. 19, 2017; 2ª Revisão em Abr. 17, 2017; Aceito em Maio 22, 2017. 
exchange and learning about the disease, also an opportunity to discuss emotional issues and caregiving difficulties. Conclusion: It is concluded that these caregivers display moderate burden and that they benefit from the support received from the Hope for ALS group. However, other strategies may be needed to minimize this burden and improve patient care safety.

Keywords: Amyotrophic Lateral Sclerosis, Caregivers, Cost of Illness, Needs Assessment.

\section{Introdução}

Doenças do neurônio motor (DNM) compõem um grupo de síndromes clínicas neurodegenerativas caracterizadas pela morte neuronal no córtex motor, tronco cerebral e corno anterior da medula espinhal. A esclerose lateral amiotrófica (ELA) é a mais comum das DNM, definida pela degeneração progressiva dos neurônios motores superiores e inferiores, causando atrofia, fasciculação, fraqueza e espasticidade. Os sinais e sintomas clínicos, únicos e padróes de progressão, não compartilhados por outras doenças, provocam mudanças consideráveis sobre o indivíduo com ELA e sua família. As limitaçôes físicas que a doença estabelece ao indivíduo o tornam incapaz de manter-se em sua vida profissional, reduzindo ainda mais sua participação social e, gradativamente, também não poderá mais ser responsável pelo seu autocuidado (SALVIONI et al., 2014).

Outros sintomas também referidos são a disartria e a disfagia devido ao comprometimento de músculos bulbares. A disfagia causará dificuldades na deglutição dos alimentos, o que gera sucessivos episódios de engasgo. Além desses, pode haver fraqueza dos músculos respiratórios, causando insuficiência respiratória, que tipicamente leva à morte do paciente. A maioria dos casos após receber o diagnóstico tem, em média, dois a cinco anos de vida. Até o momento, ainda não há tratamento curativo (PICHON et al., 2013; MATOS et al., 2011).

Estudos demonstraram que homens são mais acometidos que mulheres, numa relação de 1:1,8. Os sintomas iniciais geralmente aparecem entre 55 e 65 anos, com idade média de 64 anos. O início antes dos 30 anos, chamado de início juvenil esporádico, só é encontrado em 5\% dos casos (CALVO et al., 2014; LIMA; NUCCI, 2011).

A fraqueza muscular que acomete o paciente o faz requerer assistência quanto à mobilidade e na maioria das atividades da vida diária (AVD), de acordo com a progressão da doença. Isso exige que os profissionais proponham metas terapêuticas diversas, as quais diferirão de acordo com o estado funcional em que o paciente se encontra (LIMA et al., 2009; OLIVEIRA; PEREIRA, 2009).

A assistência da equipe multidisciplinar tem sido uma estratégia para dar melhor qualidade e prolongar o tempo de vida do paciente. A composição da equipe é variável e geralmente fazem parte dela: assistentes sociais, enfermeiros, fisioterapeuta, fonoaudiólogo, médico neurologista, psicólogo e terapeuta ocupacional. A ação desses profissionais pode beneficiar e dar conforto ao paciente e à família em diversos aspectos e estágios da doença (LIMA et al., 2009; OLIVEIRA; PEREIRA, 2009).

Ao destacar a intervenção das terapeutas ocupacionais que compóem a equipe, os encontros que foram conduzidos por elas possibilitam novas formas de se pensar o cuidado: um cuidar mais amplo e abrangente, entendendo, assim, as complexidades não somente físicas, mas contextuais, pessoais $\mathrm{e}$ singulares de cada um, visto que a atuação terapêutica ocupacional busca enfatizar as questóes holísticas e subjetivas na atençáo aos sujeitos (JOAQUIM; SILVESTRINI; MARINI, 2014).

A progressão rápida da doença torna o paciente cada vez mais dependente de um cuidador. O cuidar, mais do que um ato, é uma atitude, pois abrange um momento de atenção e zelo e constitui uma atividade de ocupação, responsabilização e envolvimento afetivo com o outro. A situaçáo-limite representada pelo cuidar de um paciente com ELA, durante o tempo de manifestação da doença, nos faz pensar em formas de redução de estresse e diminuição de sobrecarga que podem ser causadas ao cuidar do outro (FALLER et al., 2012).

Para Mello et al. (2009), as atividades de cuidado terão uma relação direta com a autonomia do paciente e questôes emocionais, como a perda de autoestima. Para o cuidador, estas podem requerer maior ou menor sobrecarga física e tempo. O cuidado ao paciente com dependência funcional torna a tarefa ainda mais exaustiva e estressante, já que estando dependente, o indivíduo necessitará de cuidados mais exigentes e por tempo indeterminado (OLIVEIRA et al., 2012; SCHNAIDER; SILVA; PEREIRA, 2009).

A ideia de sobrecarga é bastante ampla. Traduzido da língua inglesa, o termo sobrecarga do cuidador (caregiver burden) designa o estresse e se caracteriza como uma reação negativa aguda à prestação de cuidados. Emerge quando novas demandas de cuidados são introduzidas ou se intensificam e os cuidadores apresentam dificuldades para se adaptar ou modificar 
sua estratégia para atendê-las, vivenciando, assim, a sobrecarga (MANUEL et al., 2013; TOLEDO; BALLARIN, 2013).

Os cuidadores de pessoas que apresentam alguma limitação como na doença de Alzheimer, em tratamento para câncer e acamadas por grave deficiência, vivenciam mudanças no estilo de vida, como redução no tempo para se cuidar, alterações na saúde e limitação social (FALLER et al., 2012; YAMASHITA et al., 2013). Essas mudanças impactam negativamente a qualidade de vida e a satisfação pessoal no convívio familiar do cuidador, sendo percebidas também como sobrecarga (SEQUEIRA, 2010).

Os profissionais e serviços de saúde reconhecem a carga para o cuidador e assim surgem estratégias de apoio a estes, no formato de programas de atividade física e lazer; cursos de formação; grupos de apoio e alternativas para minimizar o impacto negativo sobre quem cuidam (BATISTA et al., 2012; MANUEL et al., 2013; SAMPAIO, 2011). Esses aspectos relacionados à sobrecarga e ao cuidado do cuidador justificam o interesse na realização do presente estudo.

\section{Objetivo}

Identificar a sobrecarga dos cuidadores de pessoas diagnosticadas com ELA.

\section{Método}

Trata-se de um estudo descritivo com corte transversal que busca descrever as características de determinada população ou fenômeno (GIL, 2010). A população do estudo é composta de cuidadores de pacientes com ELA, maiores de 18 anos e participantes do Grupo de Apoio denominado Esperança para ELA, que é um serviço oferecido em um hospital universitário do Recife (PE), em funcionamento há um ano. É um grupo aberto, com aproximadamente 15 cuidadores, do total dos 20 pacientes atendidos pela equipe interdisciplinar do hospital.

Os encontros, com periodicidade mensal e duraçáo de $1 \mathrm{~h} 30 \mathrm{~m}$, acontecem no Setor de Reabilitação e são conduzidos por um dos sete profissionais que compóem a equipe, de acordo com o tema a ser discutido (dois terapeutas ocupacionais, dois fisioterapeutas, uma fonoaudióloga, uma psicóloga e uma neurologista). Neles, são abordados temas preestabelecidos pelos participantes e as dúvidas que surgem durante os encontros.

Para a coleta de dados, foi aplicado um questionário sociodemográfico que identificou idade, sexo, grau de parentesco, condição de moradia com o paciente, escolaridade, renda pessoal e frequência no grupo. Também continha duas questóes abertas, em que os entrevistados opinaram sobre o grupo e os possíveis benefícios decorrentes da participação.

Para avaliar a presença de sobrecarga, foi aplicada a Escala de Sobrecarga do Cuidador - Zarit (SCAZUFCA, 2002). Trata-se de uma avaliação padronizada e validada para o Brasil por Scazufca (2002), que verifica quanto as atividades de cuidado causam impacto sobre a vida social, o bem-estar físico e emocional e as finanças do cuidador. É composta de 22 questóes afirmativas que refletem como as pessoas podem se sentir quando cuidam de outra, indicando a frequência (nunca, raramente, algumas vezes ou sempre) do sentimento, de acordo com a questáo. Cada resposta tem pontuação entre 0 e 4 pontos e o total pode variar entre 0 (zero) e 88 pontos, no qual os escores mais altos indicam maior sobrecarga. Alguns estudos adotam a classificação da pontuação descrita na Tabela 1 e esse parâmetro será usado neste estudo para atribuir o nível de estresse verificado entre os cuidadores do Grupo Esperança para ELA (MANUEL et al., 2013; OLIVEIRA et al., 2012; SCAZUFCA, 2002; WACHHOLZ; SANTOS; WOLF, 2013).

Para análise dos dados, utilizou-se estatística descritiva simples, considerando os resultados e frequências da Escala de Zarit e a pontuação geral com a respectiva classificação. Quanto aos possíveis benefícios da participação em grupo de apoio, investigados com base em questóes abertas, analisou-se o conteúdo dos discursos, de modo a agrupá-los em categorias temáticas, considerando os objetivos estabelecidos e as semelhanças e diferenças entre estes, seguindo os referenciais de análise de conteúdo de Bardin (2009).

\section{Resultados e Discussão}

A população do estudo foi formada por oito cuidadores de pacientes com ELA, maiores de 18 anos e presentes em, no mínimo, dois encontros do Grupo de Apoio a Cuidadores Esperança para

Tabela 1. Classificação da Escala de Zarit.

\begin{tabular}{cl}
\hline $\begin{array}{c}\text { Pontuação no } \\
\text { escore da Escala } \\
\text { de Zarit }\end{array}$ & \multicolumn{1}{|c}{ Nível de sobrecarga } \\
\hline $0-20$ & Ausência de sobrecarga \\
$21-40$ & Sobrecarga moderada \\
$41-60$ & Sobrecarga moderada a severa \\
$\geq 61$ & Sobrecarga severa \\
\hline
\end{tabular}

Fonte: Wachholz, Santos, Wolf (2013). Reconhecendo a sobrecarga e a qualidade de vida de cuidadores familiares de idosos frágeis. 
ELA, no período da pesquisa entre novembro e dezembro de 2014. Houve perda de participaçáo de duas cuidadoras que se encaixavam nos critérios de inclusão, porém não se conseguiu estabelecer contato durante o período referido.

O número reduzido de participantes no estudo é reflexo do período recente de constituição do grupo. Outro aspecto é a dificuldade de frequência dos cuidadores, já que muitos não conseguem ser substituídos em sua função perante o doente, para estarem presentes aos encontros mensais do grupo.

A caracterização sociodemográfica dos participantes da pesquisa está apresentada na Tabela 2 . Ao analisar, verifica-se que a populaçáo do estudo compóe-se majoritariamente de mulheres. Em relação ao grau de parentesco, em maior número são cônjuges do paciente, seguido de filhas e cunhadas.

Os achados deste estudo corroboram com os estudos de Batista et al. (2013), Borghi et al. (2013), Marques et al. (2011) e Wegner e Pedro (2010), que dizem ser composta de mulheres a maioria dos cuidadores, sendo as esposas e filhas as que configuram as cuidadoras informais ou familiares. Para a mulher,

Tabela 2. Caracterização sociodemográfica dos oito cuidadores participantes do Grupo Esperança para ELA (Recife/PE), em novembro a dezembro de 2014.

\begin{tabular}{lc}
\hline \multicolumn{1}{c}{ Variável } & Frequência \\
\hline SEXO & 1 \\
Masculino & 7 \\
Feminino & \\
IDADE & 3 \\
$\mathbf{1 8}$ a 30 anos & 4 \\
$\mathbf{4 8}$ a $\mathbf{6 0}$ anos & 1 \\
Mais de 60 & \\
PARENTESCO & 3 \\
Cônjuge & 2 \\
Filha/filho & 2 \\
Cunhada & 1 \\
Cuidadora formal & \\
ESCOLARIDADE & 1 \\
Ensino Fundamental incompleto & 3 \\
Ensino Médio completo & 3 \\
Ensino Superior completo & 1 \\
Pós-graduação & \\
RENDA FAMILIAR & 1 \\
Cuidador: gerador de renda & 2 \\
Paciente: gerador de renda & 4 \\
Cuidador com renda independente & \\
COABITA COM O PACIENTE & 5 \\
Sim & 3 \\
Não & \\
\hline
\end{tabular}

entre os vários papéis desempenhados, o de cuidadora é histórico e culturalmente evidenciado. Quando se trata de cuidar da saúde ou de quem possui alguma incapacidade, principalmente daqueles que fazem parte do círculo familiar, é a mulher que está implicitamente associada como principal cuidadora da família. A função abrange questóes relacionadas ao instinto materno e às dificuldades de alguns homens em desempenhar o cuidado, o que faz a maioria ser mulher, mesmo entre os formais (CF), que são os profissionais remunerados para realizar a atividade do cuidado.

Embora a família, de modo geral, seja também afetada pela doença, o cuidado do paciente recai, em sua maioria, sobre um único membro, que tem de assumir a responsabilidade pela prestação de assistência física, emocional e, em alguns casos, financeira, sem a ajuda de outros familiares ou profissionais (MELLO et al., 2009; YAMASHITA et al., 2013).

Isso coincide com a realidade dos entrevistados. Somente uma das famílias tem mais de um cuidador envolvido, mas o principal é claramente identificado. Nos últimos anos, o cuidador familiar tornou-se alvo de investigaçóes no Brasil e no mundo, comprovando o impacto do ato de cuidar sobre suas condiçóes de vida e saúde (BATISTA et al., 2012; BORGHI et al., 2013; MELLO et al., 2009).

Dentre os entrevistados, apenas uma se encaixa na categoria de cuidadora formal (CF). Dessa forma, náo reside com o paciente e, ao fim do seu turno de trabalho, retorna para casa. Outros dois cuidadores, embora familiares, também não residem com o paciente. Portanto, dos oito entrevistados, cinco coabitam com os pacientes, podendo ser negativo pela grande exposição aos efeitos do processo de cuidar vivenciado diariamente e, assim, gerar níveis elevados de tensão.

Estudos referem que isso ainda contribui para o surgimento de limitaçóes e modificaçóes em seu estilo de vida e para o aumento de suas atividades diárias, além de o cuidador deixar de lado sua vida particular, para assumir a vida do outro e, às vezes, responsabilizar-se também pelos demais membros da família (ANDRADE et al., 2009; GRATÃO et al., 2013; MARQUES et al., 2011).

Quanto à renda pessoal, apenas um cuidador é o único gerador de toda a renda familiar, mantendo a si, ao paciente e a sua filha, também cuidadora participante da pesquisa; dois cuidadores náo possuem nenhuma renda, tendo como suporte financeiro a renda do paciente, e quatro têm renda independente do paciente.

Dos oito participantes entrevistados, metade possui formação no Ensino Superior, três concluíram 
o Ensino Médio e apenas um não chegou a terminar o Fundamental. Uma das questốes da Escala de Zarit investiga a frequência de dúvidas dos cuidadores ao dispensar o cuidado aos pacientes e nesta, seis respondem afirmativamente. Desses, dois respondem que sempre têm dúvidas e quatro ficam em dúvida algumas vezes. Apesar de alguns estudos referirem que a baixa escolaridade pode interferir, de forma negativa, no cuidado, de acordo com os resultados, não verificamos relação entre o nível de escolaridade e as dúvidas no cuidado ao paciente (LOPES; MASSINELLI, 2013; PAULINO et al., 2009).

Também não foi possível estabelecer um padrão entre o tempo ou frequência ao grupo de apoio e as dúvidas no cuidar do paciente. A participação no grupo foi de, no mínimo, dois encontros, critério de inclusão no estudo. A participação média foi de 4,5 encontros. No entanto, entre os entrevistados, nem o nível de escolaridade, nem a quantidade de participaçóes nos encontros do grupo de apoio, pareceu influenciar a segurança em sua tarefa e cuidados prestados.

Analisando a questão na Escala de Zarit que avalia diretamente se os cuidadores se sentem, de maneira geral, sobrecarregados ao cuidar do doente (Tabela 3), verificamos que apenas um (C8) se assume como muito sobrecarregado, dois negam qualquer sobrecarga (C2 e C7) e cinco se sentem moderadamente ou um pouco sobrecarregados.

Ao relacionar a resposta dessa questão específica com a pontuação total da avaliação pela Escala de Zarit, há uma concordância entre a avaliação de sobrecarga pessoal autoinformada e a atribuída pelo escore total da escala, para os cuidadores moderadamente ou pouco sobrecarregados e para os dois que não se percebem sobrecarregados. Esses não pontuam na escala de modo suficiente para indicar presença de sobrecarga, talvez porque um deles não tem a carga afetiva da perda eminente do familiar provocada pela doença por ser cuidador formal e o outro caso é um diagnóstico recente, entâo a família ainda está fazendo os primeiros contatos com a doença e o cuidado está sendo partilhado com outros familiares.

Também destacamos que o cuidador que se percebe muito sobrecarregado é o que apresenta a pontuação mais baixa entre os que estão moderadamente sobrecarregados segundo o escore na escala, ou seja, há uma sensação pessoal mais evidente que não é informada nas demais questóes da escala. Essa percepçáo de extrema sobrecarga referida pode ser atribuída ao fato de que esse é o único homem/esposo entrevistado, sendo atualmente o único responsável pelo sustento de toda a família, agora sem o apoio da esposa que antes o auxiliava na empresa, e, hoje, os sintomas impedem a sua colaboração.

Estudos que identificam o homem e marido como cuidador (EUZÉBIO; RABINOVICH, 2006; BORGES, 2003) observam que quando é a mulher que adoece, o marido e filhos homens somente assumem a funçáo de cuidador se não houver uma mulher, geralmente uma filha, para assumir esse papel. Os autores denominam o homem, nesse caso, como "cuidado à revelia", indicando que não foi uma opção. Essa condição é explicada culturalmente pelo papel central atribuído à mulher no cuidado e ainda porque também culturalmente o homem assume o papel de provedor e é quem assume tarefas extradomiciliares, como comprar remédios, transportar para o serviço de saúde, pagar contas, convênios, entre outras.

Outro aspecto importante destacado no estudo de Borges (2003) com cuidadores de pacientes com ELA e para o qual os profissionais devem estar atentos é a qualidade anterior dos laços afetivos e familiares. A autora alerta que mesmo diante de uma doença grave e incapacitante como a ELA, a solidariedade do cuidado nem sempre é espontânea e pode ser difícil resgatar compromissos e afetos para o cuidado.

Tabela 3. Informações quanto à intensidade da sobrecarga informada pelo cuidador participante do Grupo Esperança para ELA (Recife/PE) e escore na Escala de Zarit (Recife/PE) em novembro a dezembro de 2014.

\begin{tabular}{cccc}
\hline \multirow{2}{*}{ Cuidador } & Intensidade de sobrecarga & \multicolumn{2}{c}{ Escala de Zarit } \\
\cline { 3 - 4 } & informada & Escore total & Nível de sobrecarga \\
\hline C1 & Moderadamente & 18 & Ausência de sobrecarga \\
C2 & Nem um pouco & 12 & Ausência de sobrecarga \\
C3 & Moderadamente & 46 & Moderada a severa \\
C4 & Um pouco & 27 & Moderada \\
C5 & Um pouco & 33 & Moderada \\
C6 & Moderadamente & 31 & Moderada \\
C7 & Nem um pouco & 10 & Ausência de sobrecarga \\
C8 & Muito & 26 & Moderada \\
\hline
\end{tabular}


Outra dimensão importante na relaçáo de cuidado é considerar a totalidade do ser humano que cuida, suas necessidades afetivas e até as adaptaçóes tecnológicas no espaço de cuidado domiciliar, pois a preocupação dos cuidadores vai além da realização dos cuidados corporais e mobiliza sentimentos (BRONDANI et al., 2010).

É o que avaliamos nas respostas à questão que indaga quanto ao receio do cuidador em relação ao futuro do paciente. Dos oito entrevistados, dentre os que respondem "sempre" e "frequentemente", cinco são cuidadores de pacientes com diagnóstico de ELA definida e um, de ELA possível, porém todos já apresentam sintomas bem avançados, com mobilidade prejudicada e engasgos constantes. Os dois cuidadores que respondem sentir esse receio apenas "algumas vezes" são os que cuidam de pacientes que apresentam sintomas mais "leves" que os demais e ambos ainda não apresentam mobilidade prejudicada.

O estudo de Lillo et al. (2012) relata que não existe diferença na sobrecarga dos cuidadores dos pacientes de ELA que iniciaram os sintomas pelos membros ou pelos músculos bulbares. $\mathrm{O}$ cuidador vai passando por processos de ansiedade e estresse de acordo com os impactos e privaçóes que os sintomas, que váo surgindo no paciente, causam. Com a tensão emocional, o cuidado ao paciente com ELA requer um alto esforço físico, em particular durante as fases avançadas da doença. A gravidade da doença está diretamente ligada à sobrecarga, à ansiedade e à expressão somática de depressão no cuidador (PAGNINI et al., 2010).

Quando relacionamos a gravidade do estágio em que cada paciente se encontra com as respostas dadas à questáo 8 (o sr./sra. sente que " $\mathrm{S}$ " '[o paciente] depende do sr./sra.?), verificamos que os dois que usam "sempre" e um que usa "frequentemente" como resposta são os cuidadores dos pacientes mais acometidos. Entre os demais, quatro respondem "algumas vezes" e apenas um refere "raramente". Com o avançar da doença, os pacientes requisitam mais ajuda de seus cuidadores e se tornam mais dependentes.

O estresse de conciliar as atividades de cuidado com outras é avaliado em questôes que indagam isso diretamente (questão 3) ou questionam se a presença do paciente é causa de irritação (questão 5) e tensão (questão 9) para o cuidador. Em relação ao estresse, três cuidadores afirmam "nunca" e dois respondem "raramente" se sentir dessa forma, enquanto três relatam que "algumas vezes" se sentem estressados ao conciliarem tarefas. Quanto à irritação ou à tensão, seis afirmam ausência de ambos os sentimentos e dois assumem que "algumas vezes" ou "raramente" se sentem tensos ou irritados. Isso remete ao destaque dado por Mello et al. (2009), em que antes de propor qualquer programa de suporte ou intervenção para cuidadores, é necessário conhecer as características e necessidades deles, assim como o estresse e os impactos produzidos pelo ato de cuidar.

Os cuidadores geralmente relatam algum tipo de comprometimento de saúde, como dor na coluna, enxaqueca, hipertensão, depressão, entre outros, entretanto a maioria "dificilmente" procura ajuda médica para si, por não ter com quem deixar o doente (MARQUES et al., 2011). Dos dados obtidos pelas entrevistas, apenas um cuidador responde que "frequentemente" sente não ter tempo para si pelo tempo gasto com o cuidado do paciente e ter a saúde afetada por causa do seu envolvimento com o cuidado. Os sete demais apresentam respostas que variam entre "algumas vezes", "raramente" e "nunca".

Os achados dessa pesquisa sugerem outros aspectos referidos na literatura, como o sentimento de culpa do cuidador familiar em assumir que a necessidade de cuidado seja causa de interferência em sua própria vida, dificuldade de se ausentar do lugar de cuidador principal, sentir-se recompensado emocionalmente em cuidar e ver a melhora ou gratidão da pessoa cuidada (MANUEL et al., 2013; MARQUES et al., 2011; MENDES; MIRANDA; BORGES, 2010).

Quando analisamos a questão na qual o cuidador avalia se o paciente espera que ele seja a única pessoa responsável por seu cuidado, o que representa a centralização do cuidado no cuidador, verificamos que três cuidadores informam que "sempre" é essa a expectativa do paciente, porém esses mesmos cuidadores são os que respondem que "nunca" ou "raramente" sentem sua saúde afetada ou não têm tempo para si por causa do cuidado dispensado. Com isso, parece que já conseguiram conciliar a expectativa do doente, a centralidade como cuidador e suas necessidades pessoais. Essa condição é pouco relatada na literatura.

Por fim, ao analisar a opinião dos entrevistados a respeito do grupo, a maioria atribui avaliaçáo positiva, sente-se acolhida e valoriza o serviço como um espaço importante para seu conforto pessoal, aprendizagem e facilitação no cuidado, como evidenciado a seguir:

[...] só nós, que precisamos desse apoio, podemos testemunhar o que de fato significa a doação de cada um dos integrantes (C8).

Atende todas as nossas expectativas. Acolhe e orienta (C2). 
A gente aprende com eles (profissionais) e eles com a gente (C4).

Venho com a cabeça pesada e aqui descarrego tudo (C7).

Manuel et al. (2013) e Gonçalves et al. (2011) apontam que atividades direcionadas a cuidadores, geralmente os grupos específicos, costumam ser avaliadas como importantes e que respaldam o cuidador do ponto de vista emocional, na escuta de suas queixas e acolhimento às angustias e ao estresse gerados pelo cuidado. A importância de açóes direcionadas ao cuidador também é reconhecida por seu valor instrumental, por haver possibilidades de com os profissionais e outros participantes aprender técnicas que facilitam o manejo cotidiano de situaçóes ou atos específicos do cuidado, conforme a doença exige ou progride (SAMPAIO, 2011; MELLO et al., 2009).

A análise dos benefícios de participação no grupo para si e também nas próprias atividades de cuidado resultou em cinco categorias que foram agrupadas segundo as respostas mais frequentes dos entrevistados, em que todos reconhecem benefícios em sua participação: A) orientação e adequaçáo para lidar com a doença/doente; B) segurança nos procedimentos de assistência; C) confiança no diagnóstico; D) troca de experiência e benefícios para o doente; E) benefícios para o cuidador, os quais são retratados nos fragmentos a seguir:

Batia de frente com ele e aqui fui aprendendo como lidar (C4). - Categoria A.

Sinto-me segura e orientada, para orientar a familia, que é quem convive (C2). - Categoria B.

Pude ter certeza do diagnóstico, ao ouvir os outros cuidadores falando dos sintomas, que eu também percebia nela (C6). - Categoria C.

Aprendi como agir com os sintomas, engasgos principalmente (C7). - Categoria D.

Aprendi a ter tempo pra mim também, o grupo ensina a nos cuidarmos e termos momentos de lazer"(C5). - Categoria E.

Os temas citados pelos cuidadores, agrupados nas categorias anteriores, eram discutidos durante os encontros. Além das orientaçóes para suas dúvidas ou inseguranças (categorias A e B), os cuidadores também recebiam apoio e incentivo para cuidarem da saúde, terem cuidado consigo durante o cuidar do outro e momentos para si, sem sentir culpa por isso (categorias D e E). Um dos aspectos difíceis para os cuidadores participantes era esse desligamento do cuidado, mesmo que fosse por um momento, e que ficassem tranquilos com isso, mesmo que a pessoa doente demonstrasse desaprovação.

Com isso, compreendemos que os encontros são úteis para identificar aspectos relevantes na vida do cuidador, o que pode orientar a busca de estratégias que equilibrem os aspectos alterados e favoreçam a rotina de cuidados com mais qualidade. Tal equilíbrio contribui para minimizar a associação entre exposição ao cuidado e adoecimento e auxilia a família em sua organização, para evitar a sobrecarga de apenas uma pessoa (DAHDAHA; CARVALHO, 2014).

\section{Conclusão}

Mesmo com limitaçôes pelo número reduzido de participantes no estudo, compreendemos a complexidade da tarefa de cuidar de uma pessoa com ELA, além da necessidade de avaliação da condição do cuidador. Assim, os profissionais de saúde, ao assistirem o paciente com ELA, podem estender medidas também aos cuidadores, atentando para a sua saúde física e mental, bem como maneiras que encontram ou não para enfrentar a sobrecarga advinda do ato de cuidar do outro e facilitando o cuidado ao doente, ao instrumentalizar o cuidador.

Os participantes dessa pesquisa evidenciam sobrecarga moderada em sua maioria, embora essa percepção seja contraditória em alguns aspectos mais pessoais. A aplicação da Escala de Zarit permitiu avaliar a sobrecarga e como esta se expressa em relaçáo ao tempo dedicado, à apreensão com o futuro ou à expectativa do doente. Com o estudo, também identificamos que os cuidadores se beneficiam do apoio recebido no Grupo Esperança para ELA. No entanto, outras estratégias podem ser necessárias para minimização dessa sobrecarga e segurança no cuidado ao doente.

Espera-se que os conhecimentos advindos desse estudo possam somar-se a outros e contribuir para melhorar o atendimento aos cuidadores e às pessoas com ELA.

\section{Referências}

ANDRADE, L. M. et al. A problemática do cuidador familiar do portador de acidente vascular cerebral. Revista da Escola de Enfermagem da USP, São Paulo, v. 43, n. 1, p. 37-43, 2009.

BARDIN, L. Análise de Conteúdo. Lisboa: Ediçóes 70 Lda., 2009.

BATISTA, C. M. F. et al. Sobrecarga de familiares de pacientes psiquiátricos: influência do gênero do cuidador. 
Caderno de Saúde Coletiva, Rio de Janeiro, v. 21, n. 4, p. 359-369, 2013.

BATISTA, M. P. P. et al. Repercussóes do papel de cuidador nas atividades de lazer de cuidadores informais de idosos dependentes. Revista de Terapia Ocupacional da USP, São Paulo, v. 23, n. 2, p. 186-192, 2012.

BORGES, C. F. Dependência e morte da "mãe de família”: a solidariedade familiar e comunitária nos cuidados com a paciente de esclerose lateral amiotrófica. Psicologia Estudada, Maringá, v. 8, p. 21-29, 2003. Número Especial.

BORGHI, A. C. et al. Sobrecarga de familiares cuidadores de ancianos con la Enfermedad de Alzheimer: un estudio comparativo. Revista Latino-Americana de Enfermagem, Ribeirão Preto, v. 21, n. 4, p. 1-8, 2013. Disponível em: <http://www.scielo.br/pdf/rlae/v21n4/ es_0104-1169-rlae-21-04-0876.pdf>. Acesso em: 20 ago. 2014.

BRONDANI, C. M. et al. Cuidadores e estratégias no cuidado ao doente na internação domiciliar. Texto \& Contexto Enfermagem, Florianópolis, v. 19, n. 3, p. 504510, 2010.

CALVO, A. C. et al. Amyotrophic Lateral Sclerosis: a focus on disease progression. BioMed Research International, United States, v. 2014, p. 925101, 2014.

DAHDAHA, D. F.; CARVALHO, A. M. P. Papéis ocupacionais, benefícios, ônus e modos de enfrentamento de problemas: um estudo descritivo sobre cuidadoras de idosos dependentes no contexto da família. Caderno de Terapia Ocupacional da UFSCar, Sáo Carlos, v. 22, n. 3, p. 463-472, 2014.

EUZEBIO, C. J. V.; RABINOVICH, E. P. Compreendendo o cuidador familiar do paciente com sequela de Acidente Vascular Encefálico. Temas em Psicologia, Ribeirão Preto, v. 14, n. 1, p. 63-79, 2006.

FALLER, J. W. et al. Sobrecarga e mudanças no cotidiano de cuidadores familiares de paciente com doença crônica. Ciência, Cuidado e Saúde, Paraná, v. 11, n. 1, p. 181-189, 2012.

GIL, A. C. Como elaborar projetos de pesquisa. São Paulo: Atlas, 2010.

GONÇALVES, T. R. et al. Avaliação de apoio social em estudos brasileiros: aspectos conceituais e instrumentos. Ciência \& Saúde Coletiva, Rio de Janeiro, v. 16, n. 3, p. 1755-1769, 2011.

GRATÃO, A. C. M. et al. Dependência funcional de idosos e a sobrecarga do cuidador. Revista da Escola de Enfermagem da USP, São Paulo, v. 47, n. 1, p. 137-144, 2013.

JOAQUIM, R. H. V. T.; SILVESTRINI, M. S.; MARINI, B. P. R. Grupo de mães de bebês prematuros hospitalizados: experiência de intervenção de terapia ocupacional no contexto hospitalar. Cadernos de Terapia
Ocupacional UFSCar, São Carlos, v. 22, n. 1, p. 145150, 2014.

LILLO, P.; MIOSHI, E.; HODGES, J. R. Caregiver burden in amyotrophic lateral sclerosis is more dependent on patient's behavioral changes than physical disability: a comparative study. BMC Neurology, London, v. 12, n. 156, p. 1-6, 2012.

LIMA, N. M. F. V. et al. Tradução e validação da versão brasileira da escala de gravidade na esclerose lateral amiotrófica (Egela). Fisioterapia e Pesquisa, São Paulo, v. 16, n. 4, p. 316-322, 2009.

LIMA, N. M. F. V.; NUCCI, A. Clinical attention and assistance profile of patients with amyotrophic lateral sclerosis. Arquivos de Neuro-Psiquiatria, São Paulo, v. 69, n. 2, p. 170-175, 2011.

LOPES, S. R. A.; MASSINELLI, C. J. Perfil e nível de resiliência dos cuidadores informais de idosos com $\mathrm{Al}$ zheimer. Aletheia, Canoas, n. 40, p. 134-145, 2013.

MANUEL, M. F. et al. As relaçôes familiares e o nível de sobrecarga do cuidador familiar. Escola Anna Nery Revista de Enfermagem, Rio de Janeiro, v. 17, n. 2, p. 346-353, 2013.

MARQUES, A. K. M. C. et al. Social support in the family caregiver experience. Ciência \& Saúde Coletiva, Rio de Janeiro, v. 16, p. 945-955, 2011. Suplemento.

MATOS, S. E. et al. Mortality rates due to amyotrophic lateral sclerosis in São Paulo City from 2002 to 2006. Arquivo de Neuropsiquiatria, São Paulo, v. 69, n. 6, p. 861-868, 2011.

MELLO, M. P. et al. O paciente oculto: qualidade de vida entre cuidadores e pacientes com diagnóstico de Esclerose Lateral Amiotrófica. Revista Brasileira de Neurologia, Rio de Janeiro, v. 45, n. 4, p. 5-16, 2009.

MENDES, G. D.; MIRANDA, S. M.; BORGES, M. M. M. C. Saúde do cuidador de idosos: um desafio para o cuidado. Revista Enfermagem Integrada, Ipatinga, v. 3, n. 1, p. 408-421, 2010.

OLIVEIRA, A. S. B.; PEREIRA, R. D. B. Amyotrophic Lateral Sclerosis (ALS) - three letters that change the people's life. Arquivo de Neuropsiquiatria, São Paulo, v. 67, n. 3A, p. 750-782, 2009.

OLIVEIRA, W. T. et al. Vivência do cuidador familiar na prática do cuidado domiciliar ao doente crônico dependente. Ciência, Cuidado e Saúde, Paraná, v. 11, n. 1, p. 129-137, 2012.

PAGNINI, F. et al. Burden, depression, and anxiety in caregivers of people with amyotrophic lateral sclerosis. Psychology, Health \& Medicine, Itália, v. 15, n. 6, p. 685693, 2010.

PAULINO, F. G. et al. Manejo de sintomas de demência: estratégias e participação dos cuidadores no processo de estimulação cognitiva. In: CONGRESSO IBERO-AMERICANO DE PSICOGERONTOLOGIA, 3., 
2009, São Paulo. Anais... São Paulo: Pontifícia Universidade Católica de São Paulo, 2009.

PICHON, C. E. et al. EGFR inhibitor erlotinib delays disease progression but does not extend survival in the SOD1 mouse model of ALS. PLoS One, San Francisco, v. 8, n. 4, p. 623-642, 2013.

SALVIONI, C. C. S. et al. Nutritional care in motor neurone disease/ amyotrophic lateral sclerosis. Arquivos de Neuropsiquiatria, São Paulo, v. 72, n. 2, p. 157-163, 2014.

SAMPAIO, A.S. Cuidando do cuidador: perspectiva de atuação psicológica em uma casa de apoio. Revista Argumento, Curitiba, v. 29, n. 67, p. 491-498, 2011.

SCAZUFCA, M. Versão brasileira da escala Burden Interview para avaliação de sobrecarga em cuidadores de indivíduos com doenças mentais. Revista Brasileira de Psiquiatria, São Paulo, v. 24, n. 1, p. 12-17, 2002.

SCHNAIDER, T. B.; SILVA, J. V.; PEREIRA, M. A. R. Cuidador familiar de paciente com afecção neurológica. Saúde e Sociedade, São Paulo, v. 18, n. 2, p. 284-292, 2009.
SEQUEIRA, C. A. C. Adaptação e validação da Escala de Sobrecarga do Cuidador de Zarit. Revista de Enfermagem, Coimbra, v. 2, n. 12, p. 9-16, 2010.

TOLEDO, M. O.; BALLARIN, M. L. G. S. O cotidiano de cuidadores informais de pacientes em tratamento quimioterápico. Caderno de Terapia Ocupacional da UFSCar, São Carlos, v. 21, n. 1, p. 75-81, 2013.

WACHHOLZ, P. A.; SANTOS, R. C. C.; WOLF, L. S. P. Reconhecendo a sobrecarga e a qualidade de vida de cuidadores familiares de idosos frágeis. Revista Brasileira de Geriatria e Gerontologia, Rio de Janeiro, v. 16, n. 3, p. 513-526, 2013.

WEGNER, W.; PEDRO, E. N. R. Os múltiplos papéis sociais de mulheres cuidadoras-leigas de crianças hospitalizadas. Revista Gaúcha de Enfermagem, Porto Alegre, v. 31 , n. 2, p. 335-342, 2010

YAMASHITA, C. H. et al. Associação entre o apoio social e o perfil de cuidadores familiares de pacientes com incapacidades e dependência. Revista da Escola de Enfermagem da USP, São Paulo, v. 47, n. 6, p. 1359-66, 2013.

\section{Contribuição dos Autores}

Todos os autores foram responsáveis pela concepção do texto, análise e organização de fontes, redação e revisão ao texto. A responsabilidade pela coleta dos dados foi da autora principal. Todos os autores aprovaram a versão final do texto.

\section{Notas}

${ }^{1} \mathrm{O}$ estudo foi aprovado pelo Comitê de Ética em Pesquisa da Universidade Federal de Pernambuco, CAAE $\mathrm{n}^{\circ} 38045914.0 .0000 .5208$. 